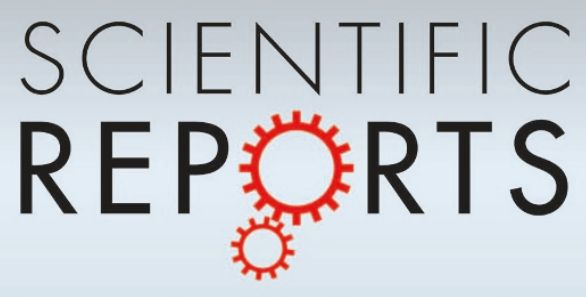

OPEN

SUBJECT AREAS:

PHASE TRANSITIONS

AND CRITICAL

PHENOMENA

KINETICS AND DYNAMICS

SURFACES, INTERFACES AND

THIN FILMS

Received

20 December 2013

Accepted

31 January 2014

Published

12 March 2014

Correspondence and requests for materials should be addressed to E.B. (erminald.bertel@ vibk.ac.at)

* Current address:

Materials Sciences

Division, Lawrence

Berkeley National Laboratory, 1

Cyclotron Road,

Berkeley, California

94720, USA.

\section{Experimental observation of defect pair separation triggering phase transitions}

\author{
M. Cordin', B. A. J. Lechner', S. Duerrbeck', A. Menzel', E. Bertel', J. Redinger² \& C. Franchini
}

'Institute of Physical Chemistry, University of Innsbruck, Innrain 82, 6020 Innsbruck, Austria, ${ }^{2}$ Institute of Applied Physics, Vienna University of Technology, Gusshausstr. 25/134, 1040 Vienna, Austria, ${ }^{3}$ Faculty of Physics, University of Vienna, Strudlhofgasse 4, 1090 Vienna, Austria.

First-order phase transitions typically exhibit a significant hysteresis resulting for instance in boiling retardation and supercooling. The hysteresis arises, because nucleation of the new phase is activated. The free-energy change is positive until the nucleus reaches a critical size beyond which further growth is downhill. In practice, the barrier is often circumvented by the presence of heterogeneous nucleation centres, e.g. at vessel walls or seed crystals. Recently, it has been proposed that the homogeneous melting of ice proceeds via separation of defect pairs with a substantially smaller barrier as compared to the mere aggregation of defects. Here we report the observation of an analogous mechanism catalysing a two-dimensional homogeneous phase transition. A similar process is believed to occur in spin systems. This suggests that separation of defect pairs is a common trigger for phase transitions. Partially circumventing the activation barrier it reduces the hysteresis and may promote fluctuations within a temperature range increasing with decreasing dimensionality.

P hase transitions are well understood from a thermodynamic point of view, but the kinetics of phase transitions is still a subject of intense research. This is particularly true of homogeneous first-order phase transitions, i.e. phase transitions originating in the bulk without nucleation at surfaces or extrinsic defects. Recently, molecular dynamics calculations with ever increasing sophistication allowed detailed insights into the atomic mechanisms triggering homogeneous melting ${ }^{1-3}$. Experiments providing similarly detailed atomic-scale information about the very beginning of the nucleation process, however, are scarce ${ }^{4}$. Some of the calculations suggest that spatial separation of defect pairs is a crucial step in early nucleation ${ }^{2,3}$. Specifically, for the homogeneous melting of ice a defect pair separation mechanism has been proposed ${ }^{3}$ which substantially reduces the free-energy barrier as compared to the purely statistical aggregation of defects until critical melting nuclei are formed. In the present study we investigate experimentally a homogeneous phase transition occurring in a twodimensional system. Observation of the phase transition with atomic resolution reveals that it, too, proceeds via a defect separation mechanism. It can be shown that the process is analogous to phase transitions in spin systems. This finding suggests that separation of defect pairs is a common mechanism for reducing the nucleation barrier thus acting as a trigger for phase transitions in a wide variety of systems. Close to the phase transition temperature $\mathrm{T}_{\mathrm{ph}}$ defect pairs are formed, split, and the propagating moieties convert one phase into the other, much like sliders in a zipper mechanism. At temperatures different from $\mathrm{T}_{\mathrm{ph}}$ the defect pairs may still be formed, but their separation is hindered by a string interaction which thus suppresses the phase transition. Since the string interaction decreases as the dimensionality of the system is reduced, quasi-critical fluctuations can occur within a temperature range which increases with decreasing dimensionality.

\section{Results}

The phase transition. Here we investigate an order-order phase transition in the $\operatorname{Br} / \mathrm{Pt}(110)$ adsorbate system at half-monolayer $(\mathrm{ML})$ coverage from a $(2 \times 1)$ rectangular phase at low temperature to a $\mathrm{c}(2 \times 2)$ checkerboard structure at room temperature. Previous analysis yielded the following results ${ }^{5-8}:$ In both ordered phases every second short bridge site on the substrate close-packed rows is occupied, but with either zero phase difference from row to row $((2 \times 1)$ structure $)$ or with a phase difference of $\pi(c(2 \times 2)$; see Figure 1$)$. The energetic preference for the $(2 \times 1)$ structure at low temperature is due to a coupling between charge density wave fluctuations associated with a periodic lattice distortion (CDW/PLD) and the Br adlayer. At low temperature the Bromine atoms lock into the CDW/PLD pattern and form a $(2 \times 1)$ structure. Since in first approximation the coupling is linear in the order parameter of the CDW, this has the effect of an external field stabilising the CDW/PLD. At room temperature, in contrast, electronic and lattice entropy lead to the disappearance ("melting") of the CDW/PLD and 

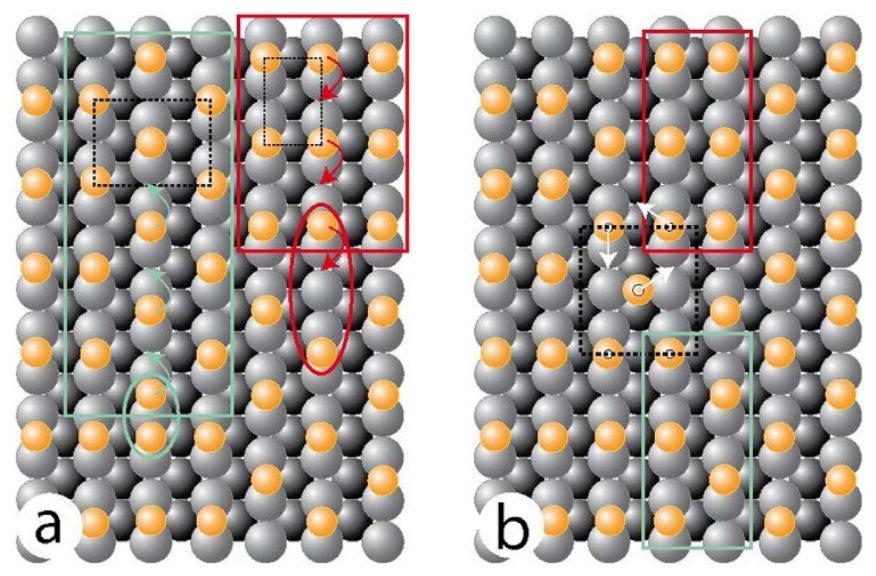

Figure 1 | Ordered structures of 0.5 ML Bromine on Pt(110): (a) Upper left and lower right: checkerboard structure with a $c(2 \times 2)$ unit cell containing 2 atoms; lower left and upper right: rectangular structure with a $(2 \times 1)$ unit cell containing 1 atom. $($ b) $(3 \times 2)$ unit cell containing 3 atoms embedded into $\mathrm{c}(2 \times 2)$ and $(2 \times 1)$ domains. Black dotted rectangles: unit cells. Also shown are possible mechanisms of checkerboard $\rightleftarrows$ rectangular conversion, i.e. by travelling solitons (turquoise ellipse) or anti-solitons (red ellipse), and via a concerted place exchange involving a $(3 \times 2)$ unit cell.

the repulsive energy in the adlayer is minimised by forming a perfectly long-range ordered quasi-hexagonal $c(2 \times 2)$ configuration on a flat substrate $6,7,9$.

Fluctuations. According to the Landau rules phase transitions between ordered states with different order parameters are always first order transitions. Nevertheless we observe in the present case quasi-critical fluctuations at $50 \mathrm{~K}$ indicating an anomalously low barrier between the two ordered states. The fluctuations are illustrated in Figure 2 showing an STM image of a Pt(110) surface covered with $0.5 \mathrm{ML}$ of $\mathrm{Br}$. The preparation shown here contains defects which allow a unique identification of the areas observed. Clearly, an inter-conversion of domains occurs in both directions. Note that in Figure 2 the length scale is set by the average defectdefect distance, but on a defect-free surface there is no preferred domain size as expected for quasi-critical behaviour.

The defect-pair mechanism. In order to investigate the mechanism for the fluctuations on an atomic scale, we first consider a preparation which is virtually free of extrinsic point defects, such as shown in Figure 3a. If one zooms in to examine the transition
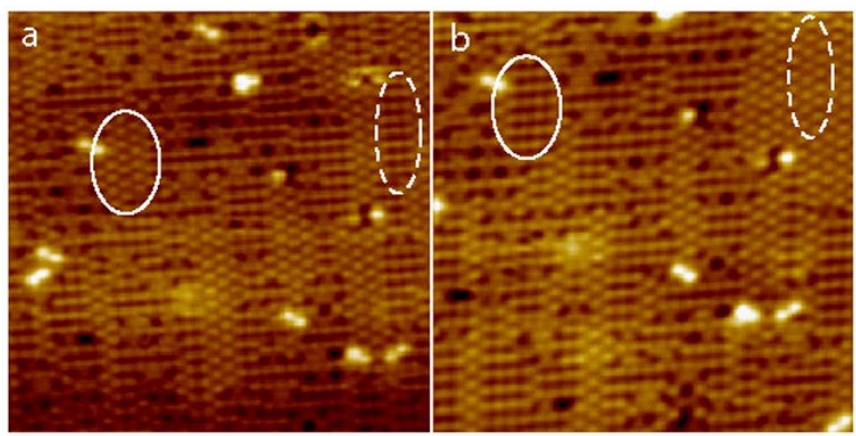

Figure $2 \mid$ STM images $\left(12.6 \times 12.6 \mathrm{~nm}^{2}\right)$ of a defective $\mathrm{Br} / \mathrm{Pt}(110)$ surface at $50 \mathrm{~K}$ recorded in succession within a time interval of about $40 \mathrm{~s}$. The Br coverage is precisely 0.5 ML. White ellipses mark identical areas where checkerboard structures were converted into rectangular ones and vice versa.

region between $(2 \times 1)$ and $c(2 \times 2)$ domains one usually observes a reduced and blurred contrast, sometimes even a loss of atomic resolution, although within the domains the atoms are well resolved. The blurring is attributable to the mobility of atoms at the domain boundaries. On careful examination, however, the transition from local $(2 \times 1)$ into $c(2 \times 2)$ domains and vice versa is revealed to be caused by either a compression (Figure $3 \mathrm{a}$ ) or a dilution (Figure $3 \mathrm{c}$ ) of $\mathrm{Br}$ atoms along the [11̄0] direction (the close-packed row direction of the substrate). Figure $3 \mathrm{~b}$ shows how such intrinsic defects are generated in an otherwise perfectly ordered domain by a density fluctuation along the $[1 \overline{1} 0]$ direction. In the row marked by white lines a displacement of a $\mathrm{Br}$ atom from its regular short bridge adsorption site towards one of its neighbours is found. This creates a dilution followed by a compression in the $\mathrm{Br}$ atom density along the row. The defect pair can be considered as a combination of soliton and anti-soliton, respectively, since each component introduces an equally large phase difference $\pi$, but with opposite sign, between neighbouring rows. If soliton and antisoliton are formed in a $\mathrm{c}(2 \times 2)$ domain and separate from each other, then, like sliders in a zipper, they leave a stripe of $(2 \times 1)$ phase with a width of three adjacent rows in their wake. Conversely, if they are formed in a $(2 \times 1)$ domain and separate, they create a $\mathrm{c}(2 \times 2)$ domain. For a soliton (dilution) or an anti-soliton (compression) to travel along the rows, only successive single-atom jumps are required as illustrated in Figure 1a.

Extrinsic defects. The presence of extrinsic defects changes the mechanism for the phase transition substantially. As seen in
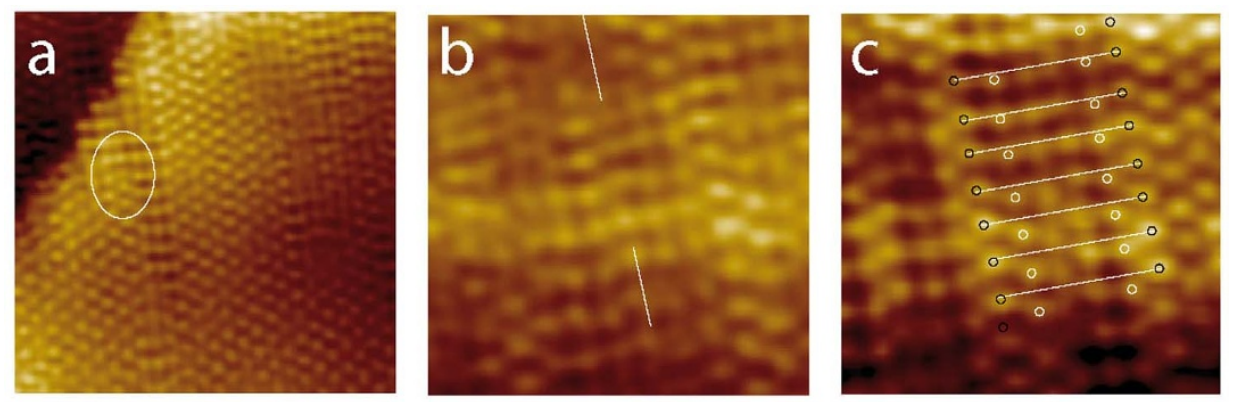

Figure $3 \mid$ Checkerboard-rectangular domain boundaries in a $0.5 \mathrm{ML} \mathrm{Br} / \mathrm{Pt}(110)$ preparation virtually free of extrinsic point-defects. (a) At the centre of the white ellipse a $\mathrm{c}(2 \times 2) \rightarrow(2 \times 1)$ transition occurs via a compression of the $\mathrm{Br}$ - Br distances along the [11̄0] direction. (b) In the row marked by white lines a $\mathrm{Br}$ atom is seen to be momentarily displaced towards its top neighbour creating a density fluctuation which consists of a compression (soliton) paired with a dilution (anti-soliton). (c) Transitions between $\mathrm{c}(2 \times 2)$ and $(2 \times 1)$ structures via anti-solitons. Black circles mark Br atoms with the regular succession of two Pt nearest-neighbour distances. White circles mark Br atoms with slightly larger distances thus accumulating a phase shift of $\pi$ from bottom to top. 


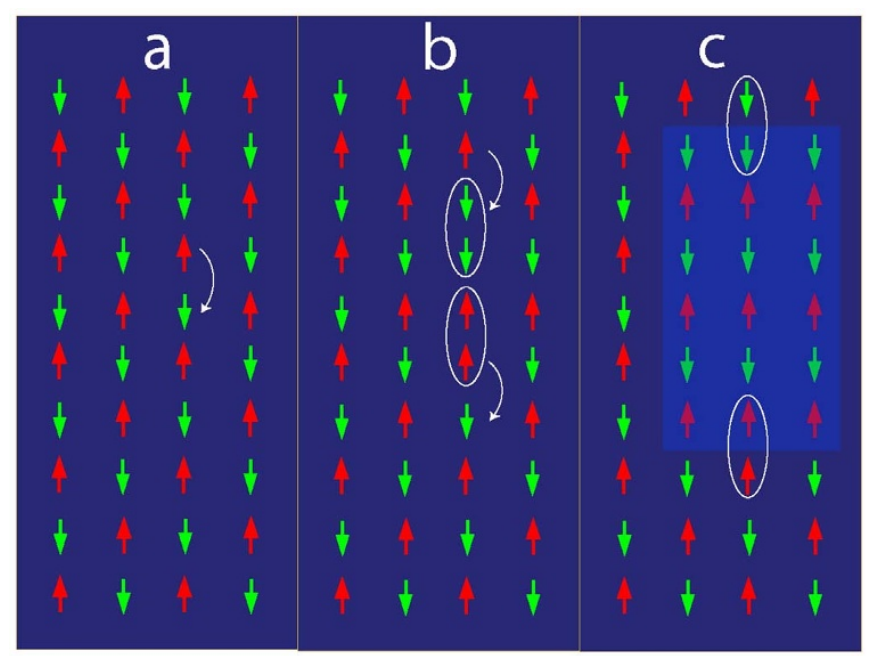

Figure $4 \mid$ Schematic of the zipper mechanism converting a checkerboard into a rectangular phase as observed in the present surface system. Red and green arrows, respectively, designate the pseudospin, which is defined as $+1 / 2$ for occupied and $-1 / 2$ for unoccupied short-bridge sites. White arrows indicate the hopping of $\mathrm{Br}$ atoms. White ellipses mark solitons and anti-solitons (double particles and double vacancies, respectively).

Figure 2, transitions between the two domains with different order parameter are in this case always associated with local $(3 \times 2)$ unit meshes (see Figure 1b) where in addition to short-bridge sites one long bridge site per unit cell is occupied. On average the $(3 \times 2)$ arrangement is only $18 \mathrm{meV}$ per $\mathrm{Br}$ atom less favourable than the $\mathrm{c}(2$ $\times 2)$ order, but - as for the $(2 \times 1)$ phase - only if the substrate is allowed to relax into a particular buckling pattern. Local nucleation of the $(3 \times 2)$ pattern is therefore associated with an energetic barrier higher than the value above, since the latter refers to a global periodic elastic distortion of the substrate. The nucleation barrier is apparently significantly higher than for the $(2 \times 1)$ buckling. In the presence of extrinsic point defects, however, the barrier is reduced and spontaneous nucleation of $(3 \times 2)$ structural elements is observed. Once they are nucleated, the $(3 \times 2)$ structure units play a dominant role in the inter-conversion of the $\mathrm{c}(2 \times 2)$ and the $(2 \times$ 1) phase, as can be concluded from a detailed inspection of Figure 2. The mechanism is sketched in Figure $1 \mathrm{~b}$ : The $(3 \times 2)$ unit mesh can propagate along the $[1 \overline{1} 0]$ direction via a concerted hopping of three neighbouring atoms in a Ring-a-Ring-o' -Roses round dance fashion. As the $(3 \times 2)$ unit mesh is shifted along the close-packed row direction the inter-conversion of the $c(2 \times 2)$ and the $(2 \times 1)$ phase proceeds.

\section{Discussion}

Introducing the occupation numbers $\pm 1 / 2$ for occupied and unoccupied short-bridge sites, respectively, i.e. assigning a pseudospin to each short bridge site, allows mapping of the present problem onto a spin system. This is shown in Figure 4 for a $c(2 \times 2) \rightarrow(2 \times 1)$ conversion. A density fluctuation translates into a pair of flipped spins (Figure 4a). This creates a soliton anti-soliton defect pair (Figure 4b). The defect moieties separate and travel independently through the system thereby creating via a zipper mechanism a stripe of the complementary phase between them. Far away from the phase transition temperature $\mathrm{T}_{\mathrm{ph}}$ appearance of the "wrong" phase raises the free energy of the system. This amounts to an effective attractive interaction between the defects and is known as string interaction. As a result, soliton and anti-soliton tend to recombine which restores the original order. Close to $\mathrm{T}_{\mathrm{ph}}$ the free energy of the two phases is balanced, the string interaction disappears, and the soliton pair becomes unbound. The travelling solitons result in quasi-critical phase fluctuations.

Figure 4 emphasizes the similarity of the present mechanism to phase conversions in strongly correlated systems. For instance, removal of an electron from an antiferromagnetic system creates a spin (spinon) and charge (holon) excitation which can also be considered as a soliton anti-soliton defect pair. In a strictly one-dimensional (1D) system (Tomonaga-Luttinger-Liquid; TLL), there is no string interaction. Thus spinon and holon may always freely separate and travel independently along the chain ${ }^{10}$. In a higher-dimensional antiferromagnet the string interaction prevents spin and charge separation, much as in the present case far from $\mathrm{T}_{\mathrm{ph}}$ the soliton anti-soliton separation is prevented.

The analogy between spin and particle chains made so far is formally reflected in the equivalence between the Heisenberg and the Bose-Hubbard Hamiltonian ${ }^{10,11}$, respectively. Of course, the propagation of the quasi-particles in the present case is not coherent in contrast to these models, since the $\mathrm{Br}$ atoms are coupled to the phonon bath of the substrate. On the other hand, the hopping of the $\mathrm{Br}$ atoms is not simply a thermal diffusion process determined by the diffusion barriers along the chain on a rigid substrate. In that case one should expect considerable dynamics at room temperature, while the measurements reveal an essentially static $c(2 \times 2)$ pattern at $300 \mathrm{~K}^{7}$. In contrast, the hopping is apparently associated with thermally driven CDW/PLD fluctuations occurring at $50 \mathrm{~K}$. We have previously proposed an extended Landau model which qualitatively describes the energetics of the phase transition ${ }^{7}$ :

$$
F=\sum_{i} J s_{i} s_{i+1}+\sum_{i} g m\left(s_{i}+s_{i+1}\right)+\frac{\alpha(T)}{2} m^{2}+\frac{\lambda}{4} m^{4}
$$

Here, $\mathrm{F}$ is a free-energy density. $m$ is the order parameter of the CDW/PLD in the substrate and the last two terms are the customary Landau expansion of the free energy in terms of the order parameter for the CDW phase transition. $J>0$ is the repulsive energy between $\mathrm{Br}$ atoms on neighbouring short-bridge sites across the rows (i.e. nnn short-bridge sites). $s_{i}, s_{i+1}$ are the corresponding occupation numbers (pseudo spins), as described above. $g<0$ is a coupling constant accounting for the coupling between the adsorbate atoms and the $\mathrm{CDW}$ in the substrate. Due to the adsorbate-substrate coupling $g$ the $\mathrm{Br}$ atoms are dragged along ${ }^{7}$ with the CDW fluctuations, i.e. fluctuations of the order parameter $m$ which occur around the CDW phase transition temperature. With increasing temperature the fluctuations become faster and the atomic resolution is $\operatorname{lost}^{5}$. As T exceeds $200 \mathrm{~K}$, however, the CDW/PLD fluctuations die out, since $m \rightarrow 0$ and, owing to the repulsive $\mathrm{Br}-\mathrm{Br}$ interaction represented by the first term in equation $(1)$, a static $\mathrm{c}(2 \times 2) \mathrm{Br}$ pattern develops. Association of the Br mobility with CDW fluctuations is supported by DFT calculations, showing that on a rigid substrate there exists a substantial barrier of the order of $1.2 \mathrm{eV}$ for the occupation of neighbouring short-bridge sites ${ }^{12,13}$.

In summary, an atomically resolved observation of a first-order phase transition in a 2D system reveals a defect-pair formation as the crucial element initiating the transformation. Close to the transition temperature the composite defect can decay into a compression (soliton) and a dilution (anti-soliton) travelling independently through the $2 \mathrm{D}$ crystal. In a kind of zipper mechanism they create a stripe of the altered phase in their wake. Far from the transition temperature the defect pair is bound by an effective string interaction. Since the string interaction decreases with dimensionality, a strongly anisotropic, quasi-1D system such as the present one can exhibit quasi-critical fluctuations in a comparatively wide temperature range around $\mathrm{T}_{\mathrm{ph}}$ even though the phase transition described by equation (1) is weakly first-order. The soliton anti-soliton zipper mechanism partially circumvents the conventional nucleation barrier. The mechanism reported here parallels similar processes in correlated systems and also resembles a recently postulated mech- 
anism for ice melting ${ }^{3}$. Therefore it is apparently of general relevance and one may expect similar zipper mechanisms to be uncovered in a wide variety of systems, once phase transitions can be observed with atomic resolution.

\section{Methods}

Experimental. Our model system has been described previously ${ }^{9,12,13}$. Almost defectfree $\mathrm{c}(2 \times 2)-\mathrm{Br} / \mathrm{Pt}(110)$ surface preparations were obtained in a preparation chamber with base pressure of $7 \times 10^{-11}$ mbar. Deposition of a few monolayers of $\mathrm{Br}$ onto the clean $(1 \times 2)$-mr reconstructed $\mathrm{Pt}(110)$ surface was followed by annealing to about $780 \mathrm{~K}$. This results in partial $\mathrm{Br}$ desorption, lifting of the $\mathrm{Pt}(110)$ missing-row reconstruction and evolution of a long-range ordered $c(2 \times 2)$ structure as verified by low-energy electron diffraction (LEED). For STM investigations the sample was freshly prepared (no LEED check, to avoid the introduction of defects) and transferred into the STM chamber with a base pressure of $\sim 3 \times 10^{-11} \mathrm{mbar}$. Topographic images were recorded with an Omicron VT-STM featuring a He flowcryostat. Defective surfaces were prepared by using a marginally lower annealing temperature after $\mathrm{Br}$ deposition. One class of defects observed after such a preparation consists of single dark objects which we attribute to $\mathrm{Pt}$ atom defects remaining after the lifting of the $(1 \times 2)$-mr reconstruction. Other defects appeared as bright objects in the STM image. Some of them seemed to be related to the $\mathrm{H}_{2} \mathrm{O}$ background pressure (total background pressure $10^{-11}$ mbar range) and showed a tendency for island formation. We tentatively attributed them to $\mathrm{H}_{2} \mathrm{O}$ adsorbing after prolonged cooling.

Calculations. DFT calculations have been performed with the Vienna Ab-initio Simulation Package (VASP) $)^{14,15}$ using the projector augmented wave method ${ }^{16}$ for periodically repeated slabs of varying substrate thickness and adsorption geometries separated by a vacuum region of minimal $14 \AA$. In order to assess the effects of substrate thickness, adsorption geometry, and the particular functional used, we performed all calculations for three different (semi)-local functionals, $\mathrm{LDA}^{17}, \mathrm{PBE}^{18}$ and PBEsol ${ }^{19}$, which yield different Pt substrate lattice constants a, with $\mathrm{a}_{\mathrm{LDA}}(3.91 \AA)$ slightly smaller, $\mathrm{a}_{\mathrm{PBE}}(3.97 \AA)$ larger and $\mathrm{a}_{\mathrm{PBE}}$ reproducing the experimental value of $3.92 \AA$. The influence of the finite substrate thickness was judged by performing all calculations on a 9 layer thick Pt slab with Br adsorbed on one surface, and on 11 layer and 15 layer thick Pt substrates with $\mathrm{Br}$ adsorbed on both surfaces. For the latter two configurations the interactions between the two $\mathrm{Br}$ layers across the substrate was studied by using both a mirror-plane and inversion geometry configuration of the two $\mathrm{Br}$ layers. All atomic positions were fully relaxed until the largest force was below $0.01 \mathrm{eV} / \AA$. Averaging the energy difference between the $\mathrm{c}(2 \times 2)$, and $(3 \times 2)$ geometries over all functionals and slab models leads to a mean square deviation of $17 \mathrm{meV}$. This spread is rather caused by the different functionals than by the finite slab models, for which it amounts to only $7 \mathrm{meV}$.

1. Cahn, R. W. Materials science: Melting from within. Nature 413, 582-583 (2001).

2. Forsblom, M. \& Grimvall, G. How superheated crystals melt. Nat Mater 4 388-390 (2005).

3. Mochizuki, K., Matsumoto, M. \& Ohmine, I. Defect pair separation as the controlling step in homogeneous ice melting. Nature 498, 350-354, DOI:10.1038/ nature12190 (2013).

4. Siwick, B. J., Dwyer, J. R., Jordan, R. E. \& Miller, R. J. D. An Atomic-Level View of Melting Using Femtosecond Electron Diffraction. Science 302, 1382-1385, DOI:10.1126/science.1090052 (2003).

5. Doná, E. et al. Fluctuations and phase separation in a quasi-one-dimensional system. Phys. Rev. Lett. 98, 186101-186104, DOI: 10.1103/Physrevlett.98.186101 (2007)
6. Amann, P. et al. Electronically driven phase transitions in a quasi-onedimensional adsorbate system. Eur. Phys. J. B 75, 15-22, DOI:10.1140/epjb/ e2010-00026-5 (2010).

7. Cordin, M. et al. Phase transitions driven by competing interactions in lowdimensional systems. EPL 92, 26004 (2010).

8. Cordin, M. et al. Comment on "Cleavage surface of the $\mathrm{BaFe}_{2-\mathrm{x}} \mathrm{Co}_{\mathrm{x}} \mathrm{As}_{2}$ and $\mathrm{Fe}_{\mathrm{y}} \mathrm{Se}_{1-\mathrm{x}} \mathrm{Te}_{\mathrm{x}}$ superconductors: A combined STM plus LEED study" Phys. Rev. B 86, 167401 (2012).

9. Blum, V. et al. Structure of the $\mathrm{c}(2 \times 2)-\mathrm{Br} / \mathrm{Pt}(110)$ surface. Phys. Rev. B 65 , 165408-165413 (2002).

10. Giamarchi, T. Quantum Physics in One Dimension. (Oxford University Press, 2004).

11. Fukuhara, T. et al. Quantum dynamics of a mobile spin impurity. Nat Phys 9, 235-241 (2013)

12. Deisl, C. et al. $(3 \times 1)-\mathrm{Br} / \mathrm{Pt}(110)$ structure and the charge-density-wave-assisted $\mathrm{c}(2 \times 2)$ to $(3 \times 1)$ phase transition. Phys. Rev. B 69, 195405-195413 (2004).

13. Deisl, C. et al. The phase diagram of halogens on $\operatorname{Pt}(110)$ : structure of the $(4 \times 1)$ Br/Pt(110) phase. J. Phys. Condens. Matter 21, 134003 (2009).

14. Kresse, G. \& Hafner, J. Ab initio molecular dynamics for liquid metals. Phys. l Rev. B 47, 558-561 (1993).

15. Kresse, G. \& Furthmüller, J. Efficient iterative schemes for ab initio total-energy calculations using a plane-wave basis set. Phys. Rev. B 54, 11169 (1996).

16. Kresse, G. \& Joubert, D. From ultrasoft pseudopotentials to the projector augmented-wave method. Phys. Rev. B 59, 1758 (1999).

17. Perdew, J. P. \& Zunger, A. Self-interaction correction to density-functional approximations for many-electron systems. Phys. Rev. B 23, 5048 (1981)

18. Perdew, J. P., Burke, K. \& Ernzerhof, M. Generalized Gradient Approximation Made Simple. Phys. Rev. Lett. 77, 3865-3868 (1996).

19. Perdew, J. P. et al. Restoring the Density-Gradient Expansion for Exchange in Solids and Surfaces. Phys. Rev. Lett. 100, 136406 (2008).

\section{Acknowledgments}

The work has been sponsored by the Austrian Science Fund through project S9004-N02. Useful discussions with S. Diehl and M. Dalmonte as well as computer support by Vienna Scientific Cluster (VSC) are gratefully acknowledged.

\section{Author contributions}

M.C. and B.A.J.L. recorded the STM images of the phase transition. S.D. contributed to image processing and data analysis. A.M. contributed to the interpretation of the phase transition. E.B. conceived the project and drafted the paper. J.R. conducted the DFT calculations and wrote part of the paper (calculational methods). C.F. participated in the DFT calculations.

\section{Additional information}

Competing financial interests: The authors declare no competing financial interests.

How to cite this article: Cordin, M. et al. Experimental observation of defect pair separation triggering phase transitions. Sci. Rep. 4, 4110; DOI:10.1038/srep04110 (2014).

(c) (i) (2) This work is licensed under a Creative Commons Attribution-

cc) visit http://creativecommons.org/licenses/by-nc-sa/3.0 